\title{
Electrochemically Switchable Double-Gate Nanofluidic Logic Device as Biomimetic Ion Pumps
}

Ming-Yang Wu, Zhong-Qiu Li*, Guan-Long Zhu, Zeng-Qiang Wu, Xin-Lei Ding, Li-Qiu Huang, Ri-Jian Mo, Xing-Hua Xia*

State Key Laboratory of Analytical Chemistry for Life Science, School of Chemistry and Chemical Engineering, Nanjing University, Nanjing 210023, China

*Email: zhongqiuli@nju.edu.cn (Z.Q. Li); xhxia@nju.edu.cn (X.H. Xia)

\section{Table of Contents}

S1. Fabrication process and experimental setup for ion current measurement of single-gate nanofluidic device

S2. Optical image of the double-gate nanofluidic device

S3. SEM images of AAO and AAO sputtered with gold layer

S4. Transmembrane conductance dependence on the time of applying redox voltages

S5. I-V curves of AAO membrane with only Au layer deposited at one side

S6. Size of ionchannel measured by an electrophoresis method

S7. Thickness of PPy layer under different membrane states

S8. Reversibly electrochemical modulation of a single-gate nanofluidic device

S9. SEM images and I-V curves of single-gate nanofluidic devices containing PPy layers of different thicknesses

S10. Top and bottom SEM images of a double-gate nanofluidic device

S11. Rectification ratios of a double-gate nanofluidic device under four different membrane states

S12. I-V curves of a bare double-gate nanofluidic membrane under four different membrane states

S13. Effects of concentration gradient $\left(\mathrm{C}_{\mathrm{GATE}-2} / \mathrm{C}_{\mathrm{GATE}-1}: 1 \mathrm{mM} / 100 \mathrm{mM}\right)$ on the functions of three different ion transport processes 


\section{S1. Fabrication process and experimental setup for ion current measurement of a}

single-gate nanofluidic device

A single-gate nanofluidic device was electrochemically deposited using a galvanostatic method. A $30 \mathrm{~nm}$-thick Au layer was sputtered onto an anodic aluminum oxide (AAO) substrate, followed by PDMS sealing and electrochemical deposition of PPy layer (Figure S1).

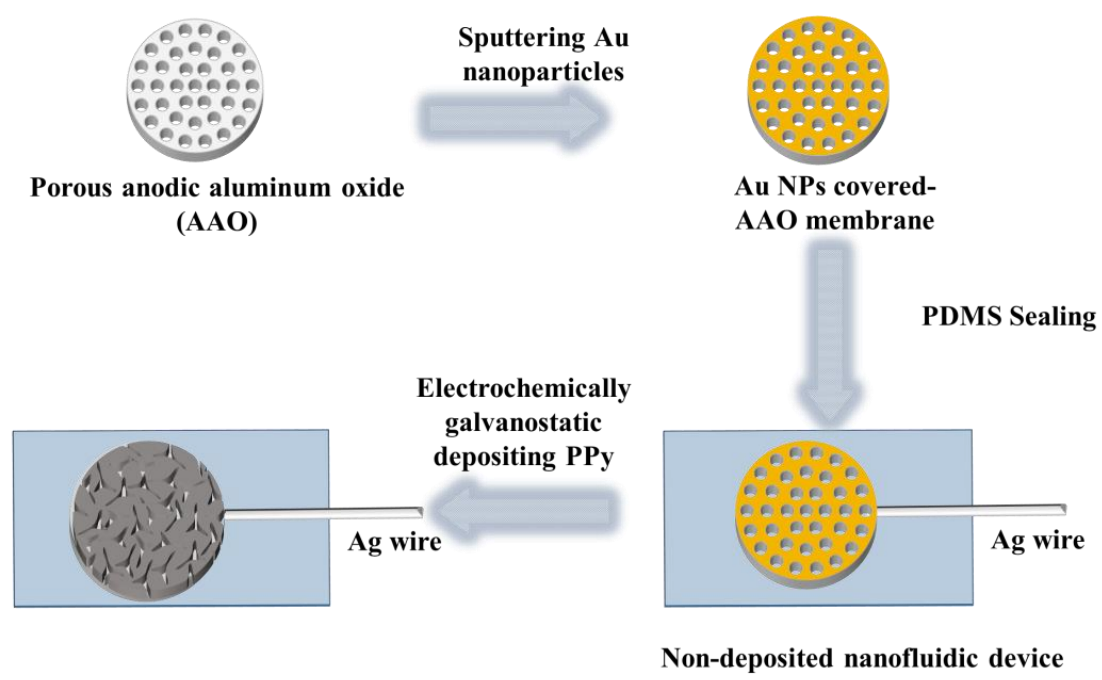

Figure S1. Fabrication procedures of a single-gate nanofluidic device.

Experimental setup for ion current measurement of a single-gate nanofluidic device.

Two half-cells filled with $100 \mathrm{mM} \mathrm{KCl}$, were connected through the single-gate nanofluidic device (Scheme S1). The enlarged scheme illustrates the electrochemical switching of the single-gate nanofluidic device between two different states. 


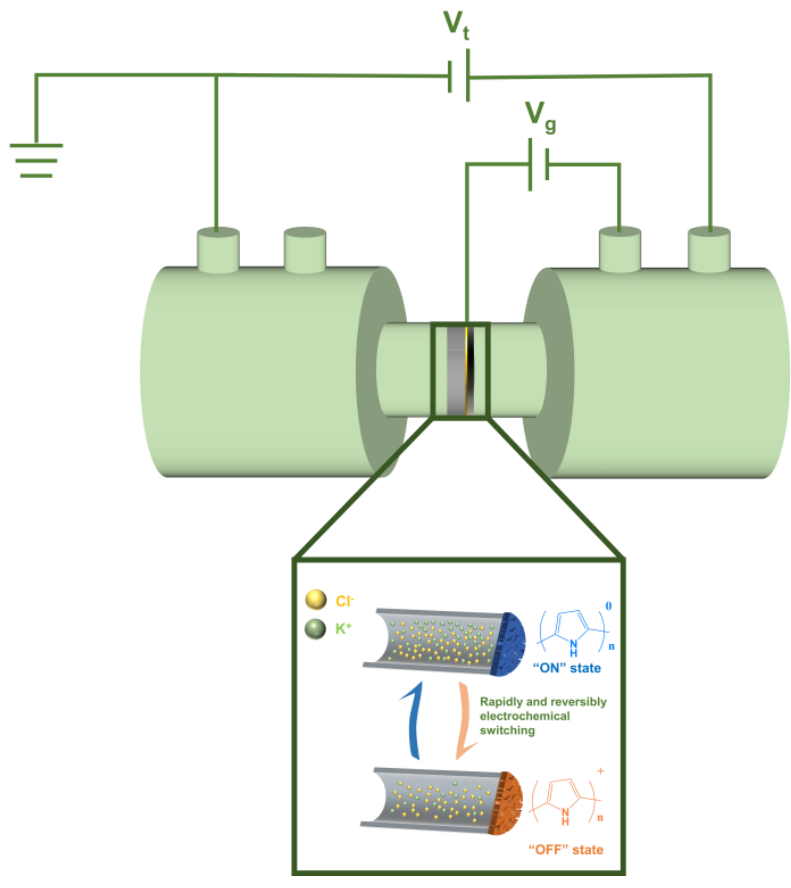

Scheme S1. Experimental setup for ion current measurement of a single-gate nanofluidic device. 


\section{S2. Optical image of the double-gate nanofluidic device}

Pyrrole monomers were electrochemically deposited on the two sides of the gold coated AAO membrane, and then sealed with PDMS sheets, resulting in two electroactive gates (Figure S2).

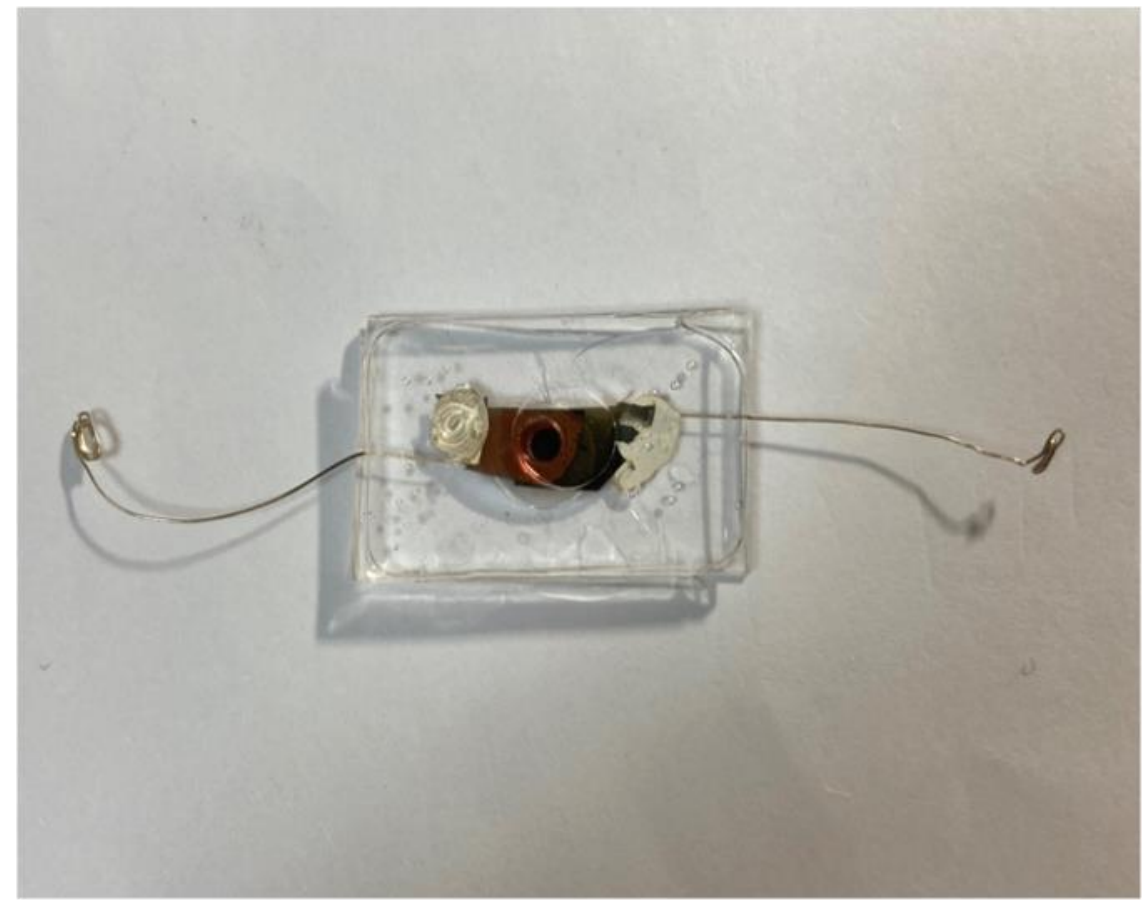

Figure S2. Optical image of the double-gate nanofluidic device. 


\section{S3. SEM images of AAO and AAO sputtered with gold layer}

The cross-sectional morphology of AAO membrane remains the same after gold deposition, exhibiting through-hole structures (Figure S3). After sputtering, a gold layer containing nanoparticles with diameter of $\sim 30 \mathrm{~nm}$ appears on the top of the membrane.
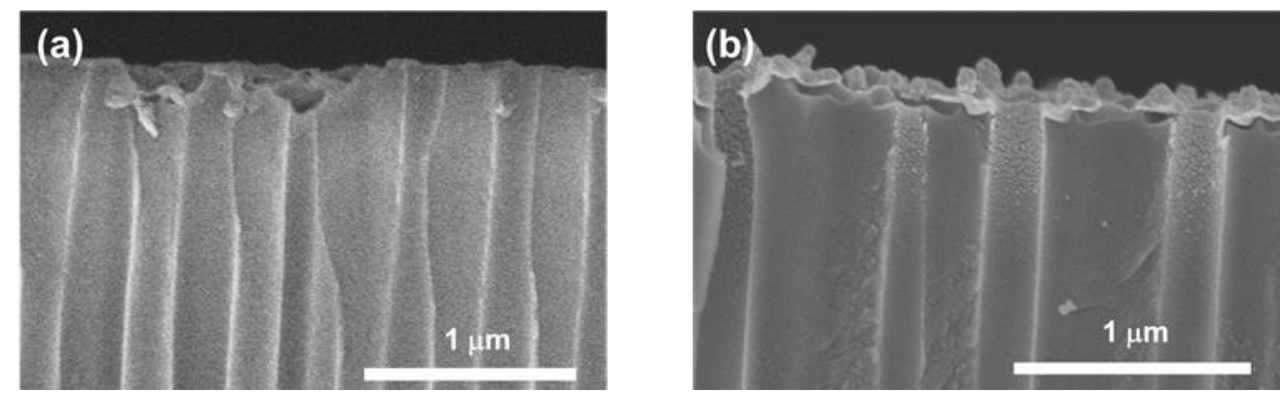

Figure S3. SEM images of AAO (a) and AAO sputtered with gold layer (b). 


\section{S4. Transmembrane conductance dependence on the time of applying redox}

voltages

As shown in Figure S4a and b, after applying reduction or oxidation voltages, the ionic conductance across the nanochannels first changes rapidly and then gradually reaches a steady state. A conversion time of $200 \mathrm{~s}$ was adopted to ensure the complete conversion between the reduction and oxidation states of PPy.
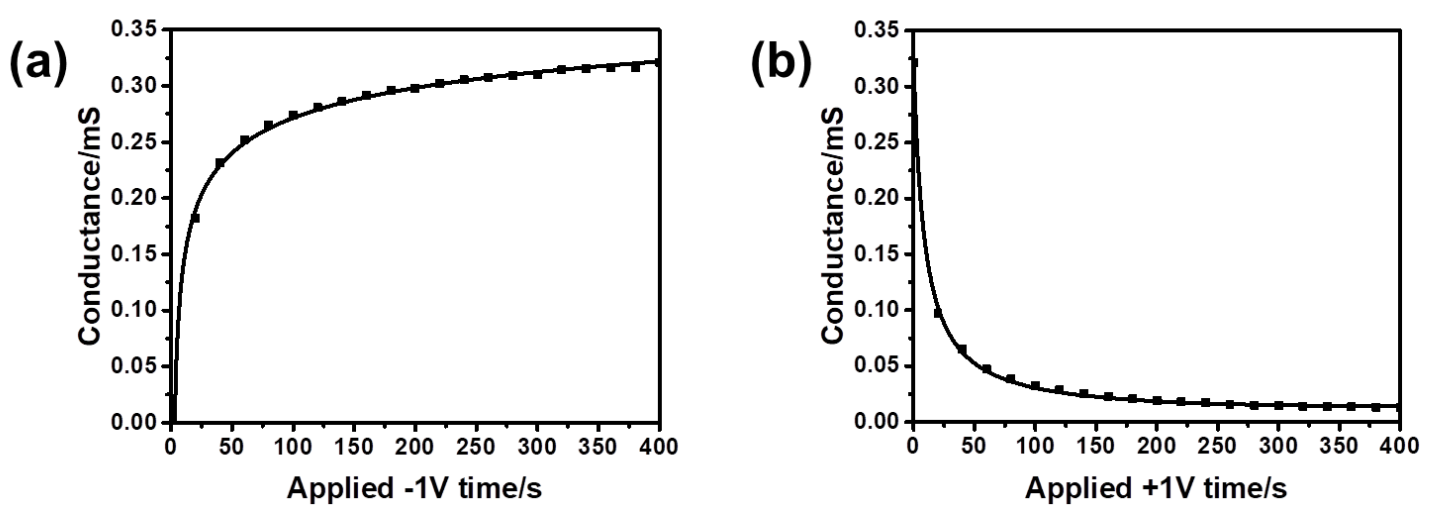

Figure S4. Changes in transmembrane conductance with time of applied gate voltage:

(a) gate voltage of $-1.0 \mathrm{~V}$; (b) gate voltage of $+1.0 \mathrm{~V}$. 


\section{S5. I-V curves of AAO membrane with only Au layer deposited at one side}

I-V curves of AAO membrane with only Au layer deposited at one side has been measured after applying different gate voltages. As shown in Figure S5, The transmembrane ionic current remained the same when switching the gate voltage from $-1 \mathrm{~V}$ to $+1 \mathrm{~V}$.

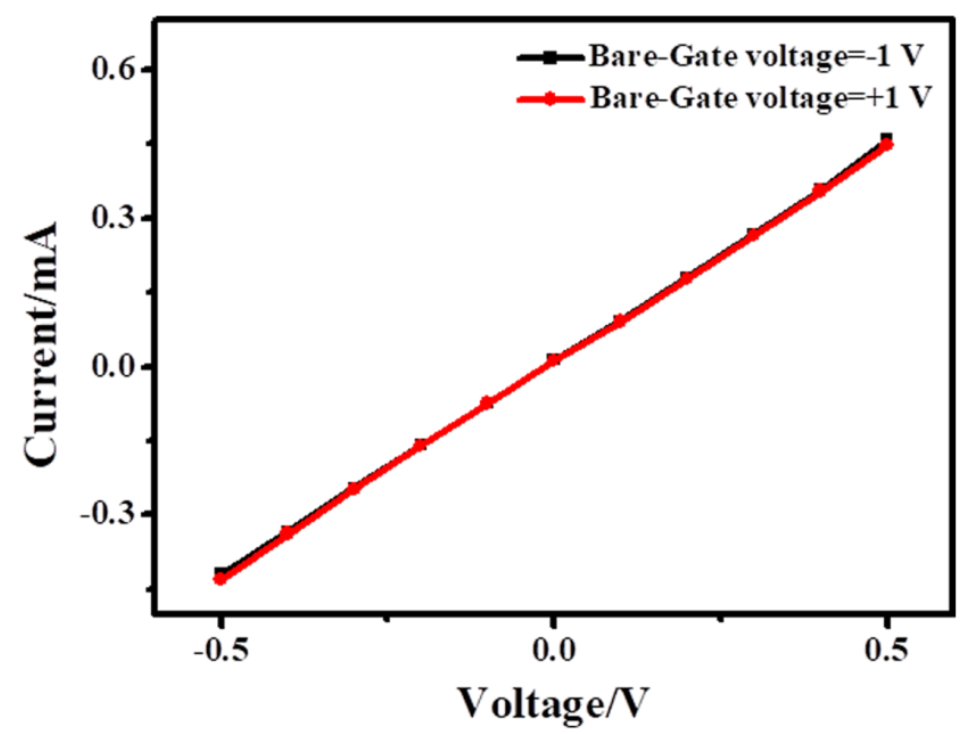

Figure S5. I-V curves of an AAO membrane with only Au layer deposited at one side. 


\section{S6. Size of ionchannel measured by an electrophoresis method}

The ionchannel size was measured by an electrophoresis method. The single-gate nanofluidic membrane was mounted between two half-cells, with $0.5 \mathrm{M} \mathrm{KCl}$ containing $10 \mathrm{mM} \mathrm{K}{ }_{3} \mathrm{Fe}(\mathrm{CN})_{6}$ in the feed cell and $0.5 \mathrm{M} \mathrm{KCl}$ in the permeate cell. Two Pt electrodes were separately put in the feed and permeate cells, and a transmembrane voltage of $-2 \mathrm{~V}$ was applied to driven $\mathrm{Fe}(\mathrm{CN}) 6^{3-}$ ions from the feed cell to the permeate cell for 30 min (Figure S6a). After then, the $\mathrm{Fe}(\mathrm{CN})_{6}{ }^{3-}$ ions in both the feed and permeate cells were characterized using cyclic voltammetry (CV). The voltage was scanned from $0 \mathrm{~V}$ to $0.5 \mathrm{~V}$ at $50 \mathrm{mV} \mathrm{s}^{-1}$ with an Au electrode as the working electrode, an $\mathrm{Ag} / \mathrm{AgCl}$ electrode as the reference, and a platinum wire as the counter electrode. As shown in Figure S6b, no redox peaks of $\mathrm{Fe}(\mathrm{CN}) 6^{3-}$ ions can be observed in the permeate cell, which indicates that $\mathrm{Fe}(\mathrm{CN}) 6^{3-}$ ions cannot transport through the PPy layer. Considering that the ionchannels inside the PPy layer is either neutral (reduction state) or positively charged (oxidation state), the electrostatic repulsion effect can be ruled out and the hindered transport of $\mathrm{Fe}(\mathrm{CN})_{6}{ }^{3-}$ ions is mainly caused by the size exclusion effect. Therefore, it is reasonable to conclude that the ionchannel size is smaller than the hydrated radius of $\mathrm{Fe}(\mathrm{CN}) 6^{3-}(0.7 \mathrm{~nm})$.

Considering that the hydrated diameter of $\mathrm{Fe}(\mathrm{CN}) 6^{3-}$ is $0.7 \mathrm{~nm}$, the ionchannel inside the PPy layer is smaller than $0.7 \mathrm{~nm}$.

(a)

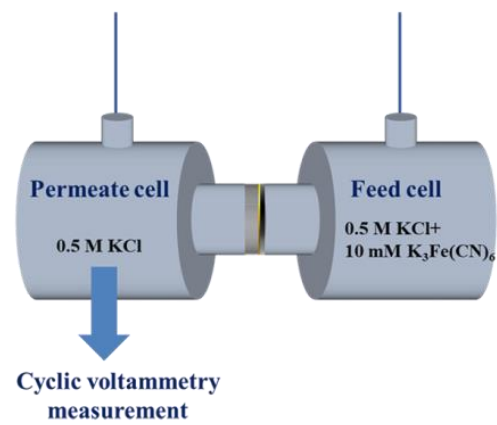

(b)

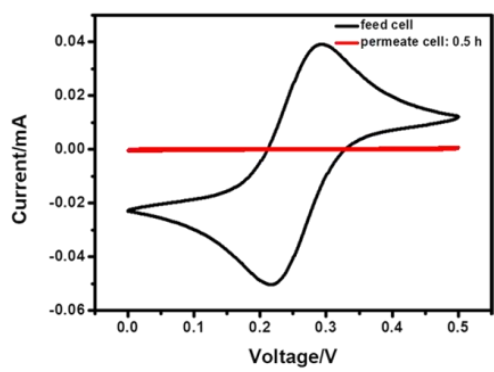

Figure S6. (a) Setup for the electrophoresis experiment. The solution in the feed cell contains $0.5 \mathrm{M} \mathrm{KCl}$ and $10 \mathrm{mM} \mathrm{K}_{3} \mathrm{Fe}(\mathrm{CN})_{6}$, and the solution in the permeate cell contains $0.5 \mathrm{M} \mathrm{KCl}$. (b) CVs of the electrolytes in permeate (red curve) and feed cells 
(black curve) in final condition. The scan rate is $50 \mathrm{mV} \mathrm{s}^{-1}$. 


\section{S7. Thickness of PPy layer under different membrane states}

A PPy layer was electrochemically deposited on Au coated Si wafer. The thickness change of the PPy layer during electrochemical conversion was measured using an atomic force microscope (AFM) (Figure S7). The thickness of the PPy layer at oxidation state was $540 \mathrm{~nm}$, which is almost the same as the one at reduction state (542 nm). The constant thickness indicates a constant ionchannel size of the PPy layer during its electrochemical conversion.
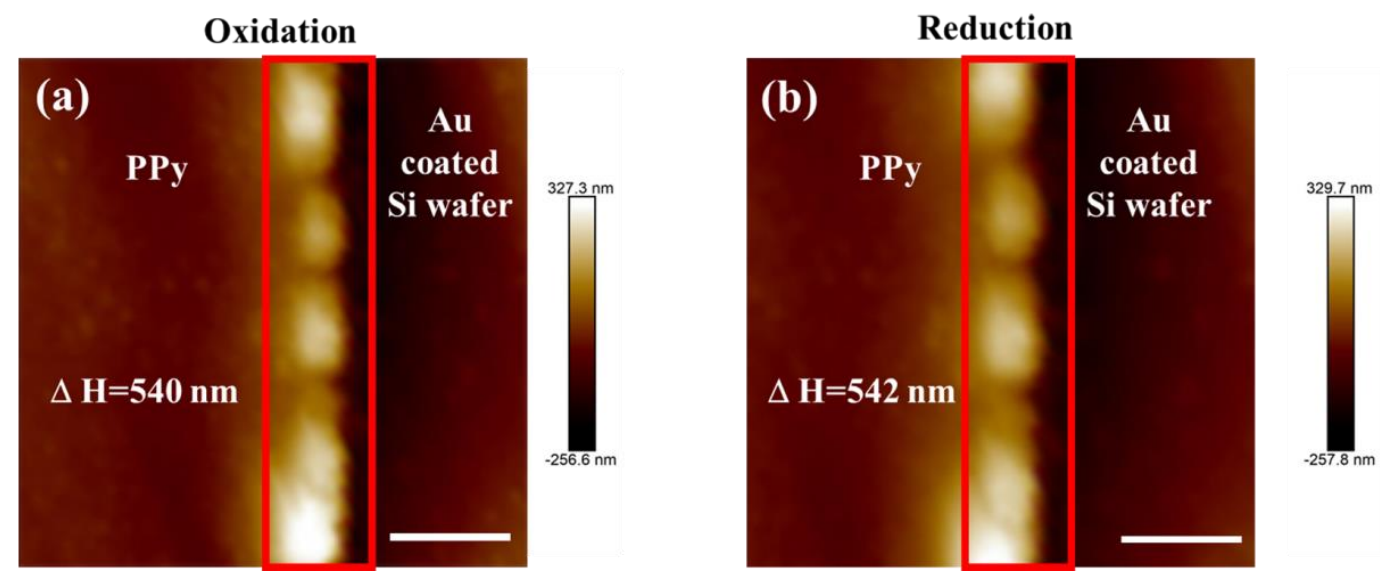

Figure S7. AFM images of the PPy layer deposited on a gold-coated Si wafer under oxidation (a) and reduction states (b). Scale bar: $3 \mu \mathrm{m}$. 


\section{S8. Reversibly electrochemical modulation of a single-gate nanofluidic device}

Reversible switching of the transmembrane conductance was observed when alternatively applying oxidization and reduction gate voltages, which indicates a good reversibility of the electrochemical modulation of the ion transport of the single-gate nanofluidic device (Figure S8).

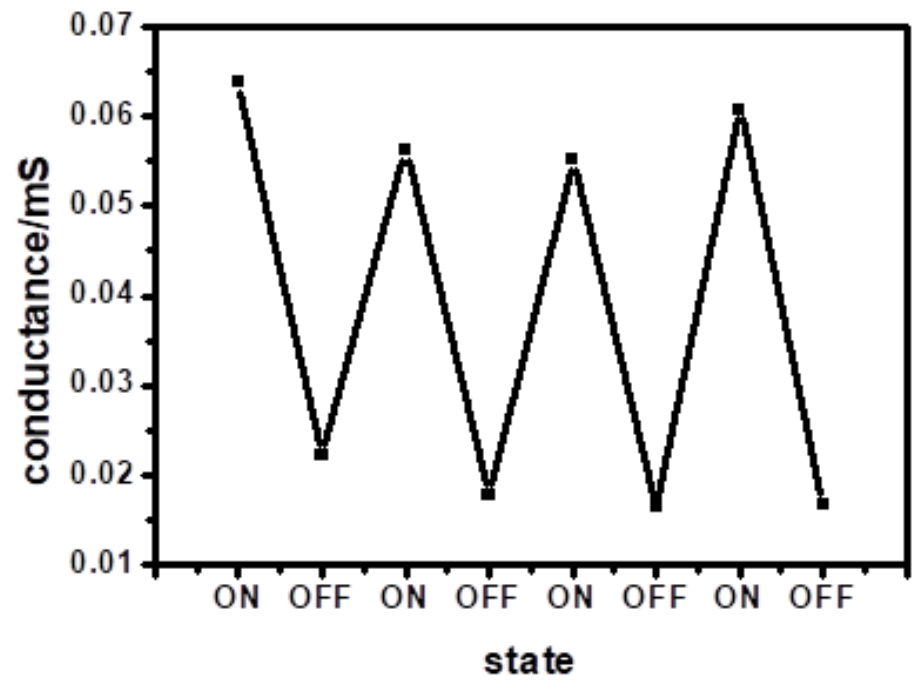

Figure S8. Reversibly electrochemical modulation of the conductance of a single-gate nanofluidic device. 


\section{S9. SEM images and I-V curves of single-gate nanofluidic devices containing PPy}

\section{layers of different thicknesses}

As shown in Figure S9 a and b, the thicknesses of the PPy layers were $200 \mathrm{~nm}$ and $400 \mathrm{~nm}$ when setting the deposition time as $50 \mathrm{~s}$ and $150 \mathrm{~s}$, respectively. The I-V curves of the oxidation and reduction states overlap for PPy layers with thicknesses of both $200 \mathrm{~nm}$ and $400 \mathrm{~nm}$, indicating no electrochemical regulation effect on ion transport (Figure 9c and d). This is because the gold-deposited AAO membrane is not fully covered by PPy film under short polymerization time and the ion transport is thus dominated by the defects and cracks.

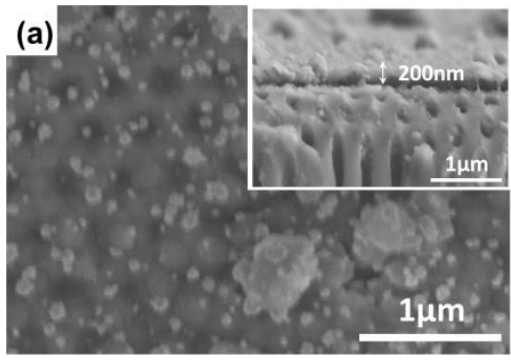

(c)

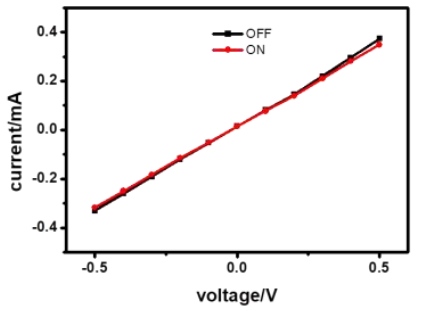

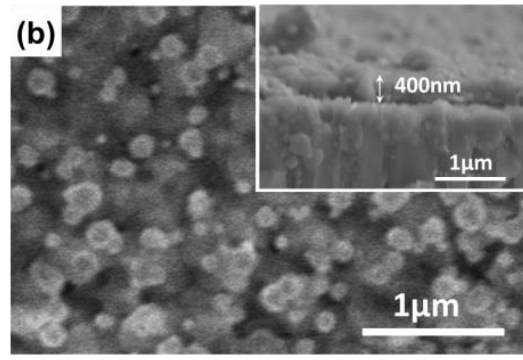

(d)

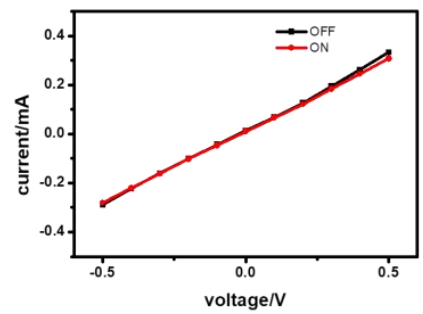

Figure S9. SEM images of the single-gate nanofluidic devices containing PPy layers fabricated with deposition time of $50 \mathrm{~s}$ (a) and $150 \mathrm{~s}$ (b), respectively. I-V curves of the single-gate nanofluidic devices containing PPy layers with thicknesses of $200 \mathrm{~nm}$ (c) and $400 \mathrm{~nm}(\mathrm{~d})$, respectively. 
S10. Top and bottom SEM images of a double-gate nanofluidic device

PPy layers on both top and bottom sides of a double-gate nanofluidic device were dense and compact (Figure S10).

(a)

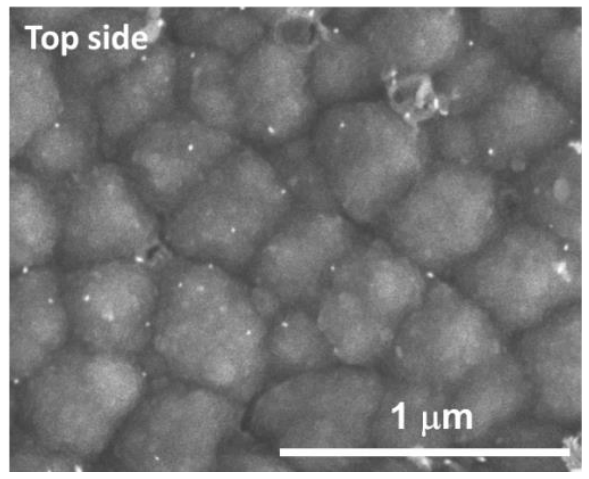

(b)

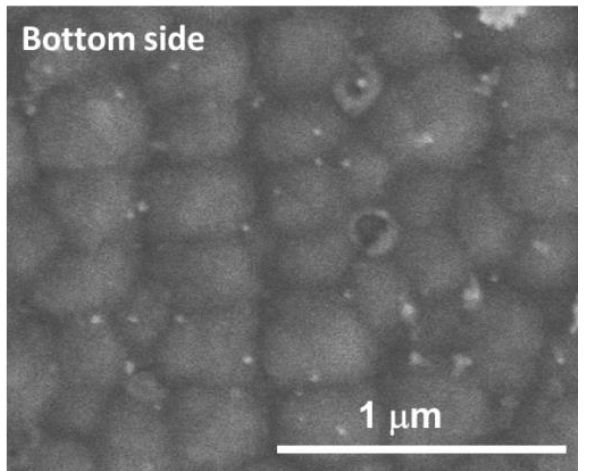

Figure S10. SEM images of a double-gate nanofluidic device: top side (a); bottom side (b). 
S11. Rectification ratios of a double-gate nanofluidic device under four different membrane states

Four I-V curves are observed by applying symmetric/asymmetric electrochemical stimuli, corresponding to four different states of ion transport, i.e. ON-ON, OFF-OFF, ON-OFF and OFF-ON. The ionic current rectification ratio, which is defined as the ratio of the absolute value of the current measured at $+1 \mathrm{~V}$ to $-1 \mathrm{~V}$ applied potentials, remains as 1 for ON-ON and OFF-OFF. However, it increases from 0.36 to 6.06 when the electrochemical stimuli changes from ON-OFF into OFF-ON (Figure S11), showing rectification inversion.

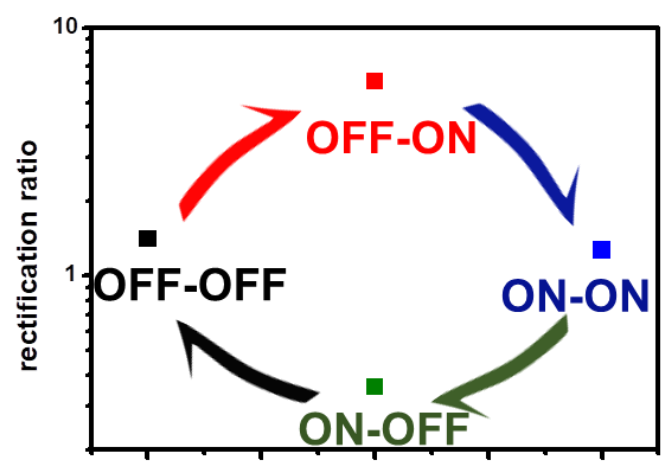

Figure S11. Rectification ratios of a double-gate nanofluidic membrane under four different membrane states. 
S12. I-V curves of a bare double-gate nanofluidic membrane under four different membrane states

The electrochemically gated ion transport was also studied using a bare device with only gold deposited on both sides of an AAO membrane. As shown in Figure S12, the I-V curves barely change when applying different gate voltages.

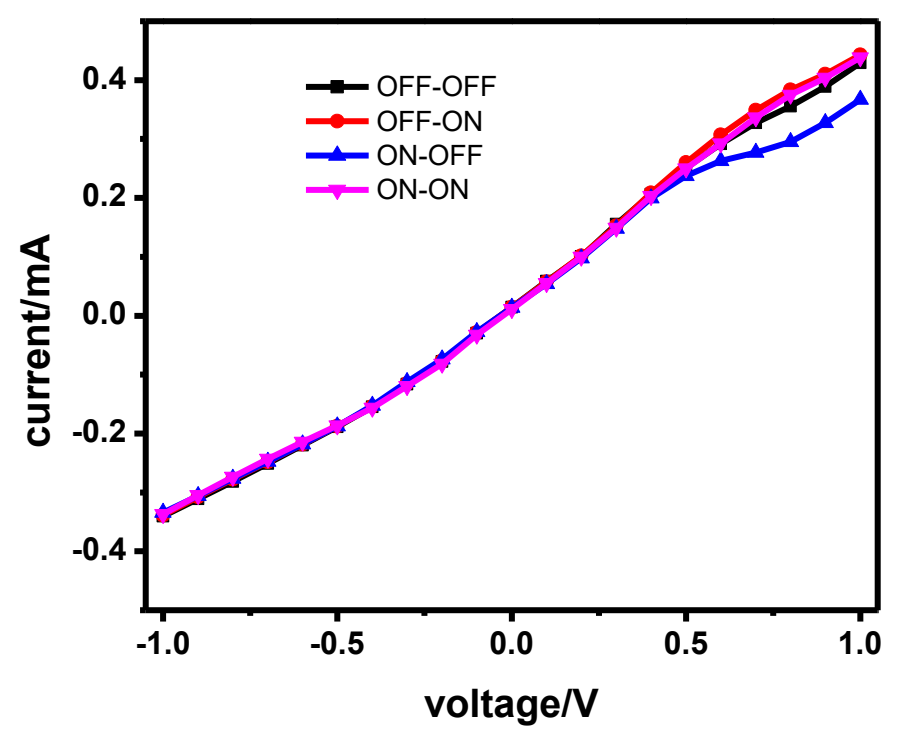

Figure S12. I-V curves of a bare double-gate nanofluidic device under four different membrane states. 
S13. Effects of concentration gradient (CGATE-2/C GATE-1:1 mM/100 mM) on the functions of three different ion transport processes

Effects of concentration gradient $\left(\mathrm{C}_{\mathrm{GATE}-2} / \mathrm{C}_{\mathrm{GATE}-1}: 1 \mathrm{mM} / 100 \mathrm{mM}\right)$ on the functions of three different ion transport processes: ion pump feature, ion channel feature and fail-safe ion pump feature (Figure S13).

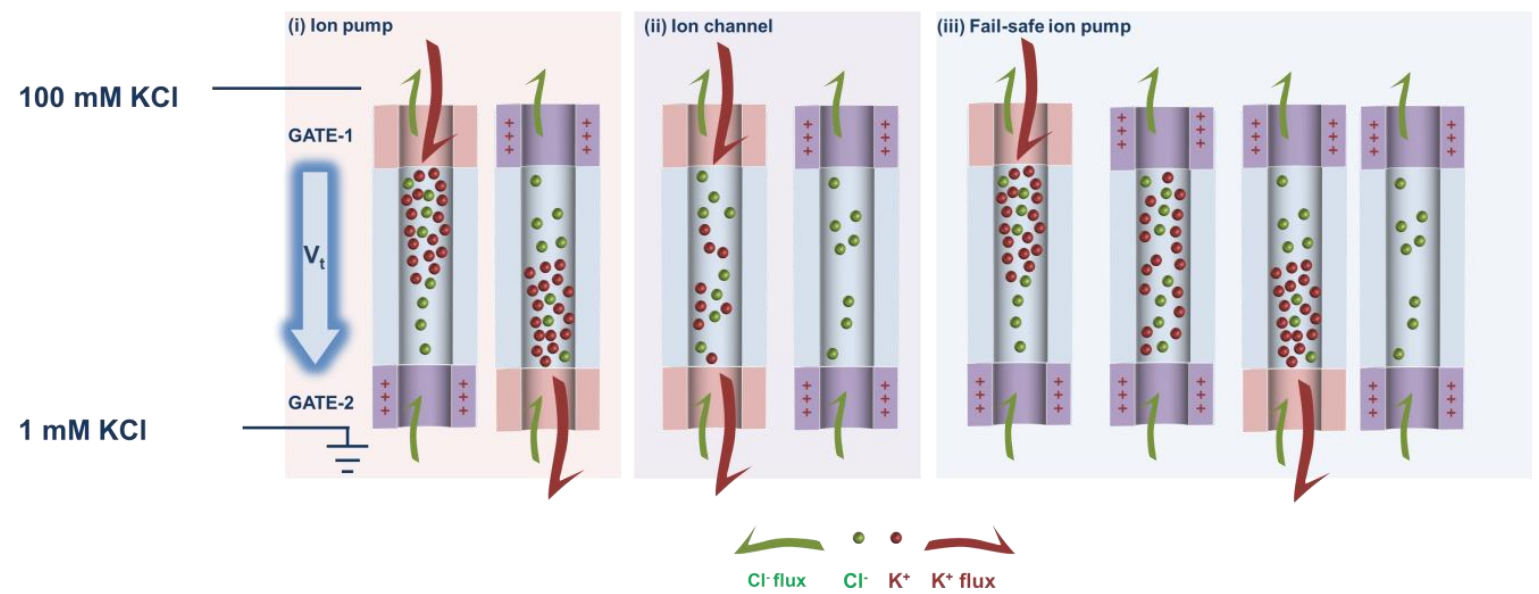

Figure S13. Effects of concentration gradient $\left(\mathrm{C}_{\mathrm{GATE}-2} / \mathrm{C}_{\mathrm{GATE}-1}: 1 \mathrm{mM} / 100 \mathrm{mM}\right)$ on the functions of three different ion transport processes. 